\title{
Pathogenic antibodies induced by spike proteins of COVID-19 and SARS-CoV viruses
}

\section{Huiru Wang ( $\nabla$ wjane60527@yahoo.com )}

Huirui Biopharma, Co., Ltd.

\section{Qiuchi Chen}

HuaAn McAb Biotechnology, Co., Ltd.

\section{Yue Hu}

Biolynx Technology (Hangzhou), Co., Ltd.

\section{Xiancong Wu}

HuaAn McAb Biotechnology, Co., Ltd.

\section{Lin Dai}

HuaAn McAb Biotechnology, Co., Ltd.

\section{Yuekai Zhang}

Biolynx Technology (Hangzhou), Co., Ltd.

\section{Fang Li}

HuaAn McAb Biotechnology, Co., Ltd.

Jinfeng Lu

Biolynx Technology (Hangzhou), Co., Ltd.

\section{Yuxing Chen}

HuaAn McAb Biotechnology, Co., Ltd.

\section{Xiaoling Liu ( $\sim$ liuxl@wizybio.com )}

HuaAn McAb Biotechnology, Co., Ltd.

\section{Research Article}

Keywords: COVID-19 infection, pathogenic antibodies, anti-spike antibodies, new mechanisms of pathogenesis

Posted Date: June 16th, 2021

DOI: https://doi.org/10.21203/rs.3.rs-612103/v2

License: (c) (i) This work is licensed under a Creative Commons Attribution 4.0 International License. Read Full License 


\title{
Pathogenic antibodies induced by spike proteins of COVID-19 and SARS-CoV
}

\section{viruses}

\author{
Huiru Wang ${ }^{1 *}$, Qiuchi Chen ${ }^{2}$, Yue $\mathrm{Hu}^{3}$, Xiancong $\mathrm{Wu}^{2}$, Lin Dai ${ }^{2}$, Yuekai Zhang ${ }^{3}$, Fang $\mathrm{Li}^{2}$, Jinfeng $\mathrm{Lu}^{3}$, Yuxing \\ Chen ${ }^{2}$, and Xiaoling Liu $^{2 *}$
}

1. Huirui Biopharma, Co., Ltd. Hangzhou, China

2. HuaAn McAb Biotechnology, Co., Ltd. Hangzhou, China

3. Biolynx Technology (Hangzhou), Co., Ltd. Hangzhou, China

\begin{abstract}
This study, using a virus-free mouse model, explores the pathogenic roles of certain antibodies specific to the spike proteins of highly pathogenic coronaviruses such as the COVID-19 and the SARS-CoV viruses. Our data showed that these pathogenic antibodies, through a mechanism of Antibody Dependent Auto-Attack (ADAA), target and bind to host vulnerable cells or tissues such as damaged lung epithelium cells, initiate a self-attack immune response, and lead to serious conditions including ARDS, cytokine release, and death. Moreover, the pathogenic antibodies also induced inflammation and hemorrhage of the kidneys, brain, and heart. Furthermore, the pathogenic antibodies can bind to unmatured fetal tissues and cause abortions, postpartum labors, still births, and neonatal deaths of pregnant mice. Novel clinical interventions, through disrupting the host-binding of these pathogenic antibodies, can be developed to fight the COVID-19 pandemic. In addition, the new concept of ADAA explored by this study may be applicable to other infectious diseases, such as the highly pathogenic influenza infections. It should be noted that the majority of anti-spike antibodies are non-pathogenic, as only 2 of 7 monoclonal antibodies tested showed significant pathogenic effects.
\end{abstract}

The pandemic of coronavirus disease 2019 (COVID-19) is a major threat to worldwide population health and economies $^{1,2}$. One week after onset of the COVID-19 infection, the clinical condition of the disease can become severe, progressing with hypoxemia and dyspnea, and rapidly develop to acute respiratory distress syndrome (ARDS) in 17\% of patients, and 65\% of these patients worsened and died due to multi-organ dysfunction $^{3,4,5}$. Similar clinical characteristics have also been observed in patients infected with other highly pathogenic respiratory viruses such as the severe acute respiratory syndrome (SARS) virus (SARS-CoV) ${ }^{6}$, the middle east respiratory syndrome (MERS) virus (MERS-CoV) ${ }^{7}$, and the avian H1N5 and H7N9 influenza viruses ${ }^{8,9}$. In some cases the viral load was low or undetectable at the time of severe illness ${ }^{10}$, suggesting that there were other causes of death than the virus alone. It has been reported that over-reacting immune responses as well as the accompanying cytokine storms played a critical role in the deaths of the infected $^{11,12,13}$, yet what initiated the cytokine storms remains a mystery. We have made the unexpected discovery in a mouse model that injection of anti-influenza sera into pregnant mice induced lung inflammation in mouse pups born to the dames ${ }^{14}$. Thus, we suspected that certain antibodies induced by a highly pathogenic virus may be pathogenic themselves, through the targeting of host cells or tissues. To prove this hypothesis, we investigated the pathogenic effects of anti-coronavirus antibodies, including anti-COVID-19 viral antibodies, in this study.

\section{Results \\ Anti-COVID-19 antibodies bound to damaged lung epithelium cells}


Table 1. Human monoclonal antibodies isolated from patients with COVID-19 infection

\begin{tabular}{lllll}
\hline \multicolumn{1}{c}{ MAb name } & Fc format & \multicolumn{1}{c}{ Binding antigen } & & NCBI crystal structure site \\
\hline B38 & human IgG1 & COVID-19 Spike Protein S1 & https://www.ncbi.nlm.nih.gov/Structure/pdb/7BZ5 & 15 \\
S309 & human IgG1 & COVID-19 Spike Glycoprotein & https://www.ncbi.nlm.nih.gov/Structure/pdb/6WPS & 19 \\
4A8 & human IgG1 & COVID-19 Spike Glycoprotein & https://www.ncbi.nlm.nih.gov/Structure/pdb/7C2L & 16 \\
CC12.3 & human IgG1 & COVID-19 Spike Protein S1 & https://www.ncbi.nlm.nih.gov/Structure/pdb/6XC4 & 20 \\
CR3022-b6 & human IgG1 & COVID-19 Spike Glycoprotein & https://www.ncbi.nlm.nih.gov/Structure/pdb/7KZB & 18 \\
REGN10987 & human IgG1 & COVID-19 Spike Protein S1 & https://www.ncbi.nlm.nih.gov/Structure/pdb/6XDG & 17 \\
REGN10933 & human IgG1 & COVID-19 Spike Protein S1 & https://www.ncbi.nlm.nih.gov/Structure/pdb/6XDG & 17 \\
\hline
\end{tabular}

The coronavirus of SARS-CoV-2 is responsible for the COVID-19 infection. Antibodies specific to the SARS-CoV-2 (COVID-19) virus and the SARS-CoV virus were tested for their binding to the human lung epithelium cell line A549. Seven of the antibodies tested were monoclonal antibodies specific to the SARS-CoV-2 spike protein. These antibodies have been previously reported by others and were isolated from the B cells of COVID-19-infected patients ${ }^{15,16,17,18,19,20}$. These seven monoclonal antibodies were reproduced according to the gene sequences encoding the antibodies, from the NCBI crystal structure site (Table 1). The other anti-coronavirus antibodies tested include polyclonal rabbit anti-SARS-CoV-2 spike one (anti-COVID-19 S1), rabbit anti-SARS-CoV spike glycoprotein (anti-SARS S), and monoclonal mouse anti-SARS-CoV nucleocapsid (N) proteins (anti-SARS $\mathrm{N})$. These antibodies were commercially available (Bioss Antibodies) and were produced by immunization of animals with related recombinant proteins of the SARS-CoV-2 and SARS-CoV viruses. The polyclonal anti-SARS-CoV-2 nucleocapsid (N) protein (anti-COVID-19 N) antibodies were obtained by immunization of rabbits with the recombinant nucleocapsid $(\mathrm{N})$ protein of SARS-CoV-2 virus (HuaAn McAb Biotechnology).
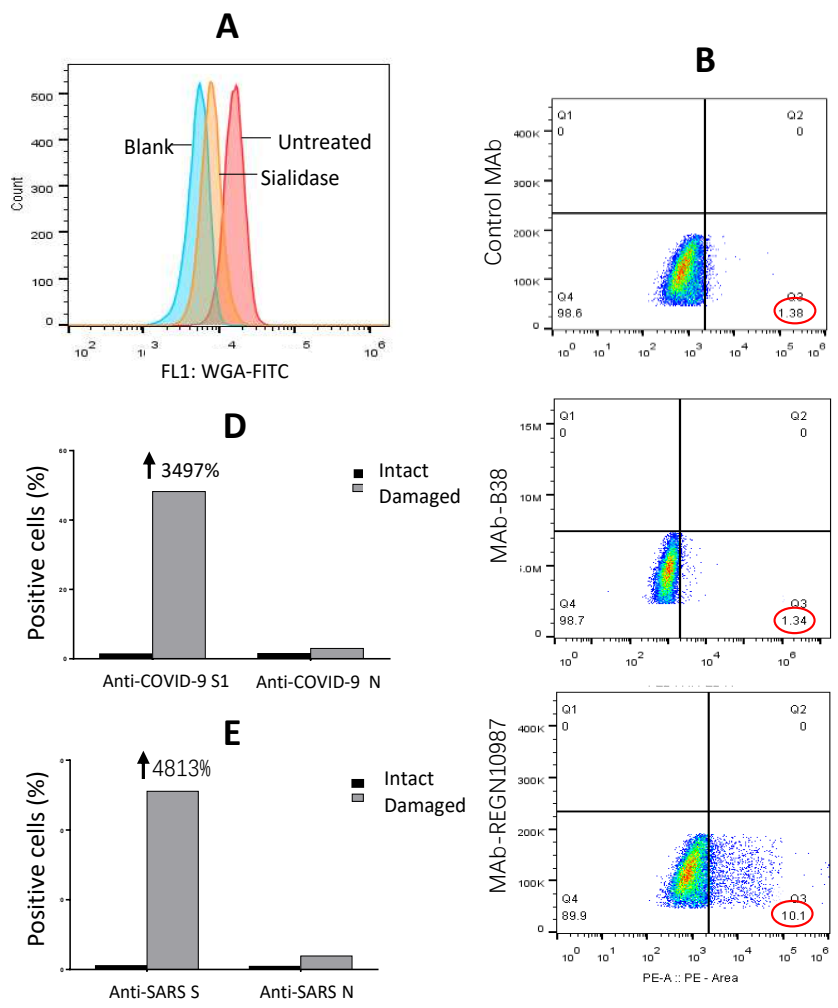
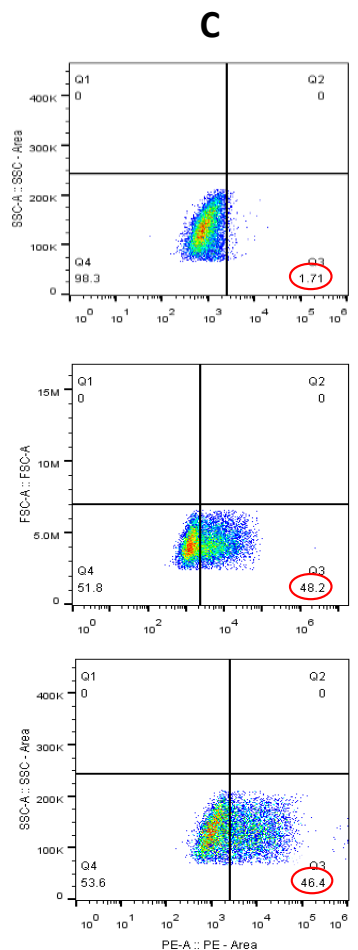

Figure 1. A flow cytometry analysis for determination of the sialic acid levels on the surface of human lung epithelium A549 cells that are either healthy (untreated) or damaged (sialidase treated) with missing sialic acid on the cell surface (A); and binding of antibodies specific to the spike $(\mathrm{S})$ or nucleocapsid $(\mathrm{N})$ proteins of COVID-19 virus and SARS virus, to the healthy (intact) or damaged A549 cells (D-E). B-C: binding of human monoclonal anti-spike antibodies B38, REGN10987, and CR3022-b6 (control $\mathrm{MAb}$ ), to healthy (B) or damaged (C) A549 cells. 
Binding of those antibodies to healthy (intact) or damaged lung epithelium cells were tested with A549 cells. In order to induce damaged cells, A549 cells were treated with neuraminidase (sialidase) according to manufacturer's instructions (Roche) ${ }^{21}$. The fluorescent labeled wheat germ agglutinin (WGA), which specifically binds to N-Acetylneuraminic acid (Vector), and a flow cytometry analysis were used to determine the levels of sialic acid on the surface of A549 cells. The damaged cells with missing sialic acid on the cell surface were used to imitate the in vivo conditions of infected lung epithelium cells (sick cells). As shown in Figure 1A, the level of sialic acid on the surface of A549 cells treated with sialidase was lower than those of untreated cells (Figure 1A).

The seven human monoclonal antibodies specific to the COVID-19 spike protein (Table 1) were tested for their binding to A549 cells. As shown in Figure 1C, two out of seven $(28.6 \%)$ such antibodies, REGN10987 ${ }^{17}$ and B38 ${ }^{15}$, strongly bound to the damaged A549 cells with missing sialic acid. REGN10987 also weakly bound to healthy A549 cells while B38 did not bind to the healthy A549 cells (Figure 1B). The control antibody of CR3022-b6 ${ }^{20}$ did not bind to the healthy A549 cells nor the damaged cells (Figure 1B and 1C). The other four human monoclonal antibodies bound neither significantly to healthy A549 cells nor to damaged cells. Further, the antibodies specific to the spike glycoprotein of SARS-CoV virus (anti-SARS S) strongly bound to the damaged A549 cells missing sialic acid, but not to the healthy A549 cells with sialic acid (Figure 1E). In addition, the polyclonal antibody specific to the nucleocapsid (N) protein of COVID-19 virus (anti-COVID-19 N) and the antibody specific to the nucleocapsid protein of SARS-CoV virus (anti-SARS N) bound neither significantly to the healthy A549 cells nor to the damaged cells (Figure 1D and 1E). Similar results were also observed with kidney embryonic cells of HEK-293 (data not shown).

Taken together, the results of the in vitro assay indicated that certain antibodies specific to the spike proteins of the COVID-19 and SARS-CoV viruses have the potential to mislead the immune system to attack the host by binding to sick cells such as human lung epithelium cells in vivo. We termed this mechanism of action of the antibodies as "Antibody Dependent Auto-Attack (ADAA)". The antibody of REGN10987 may have a higher ADAA potential by activating immune responses in vivo since it binds not only to sick cells but also to healthy cells, albeit at a lower rate.
A

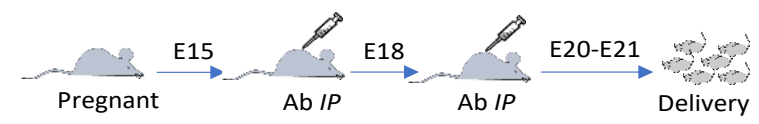

B
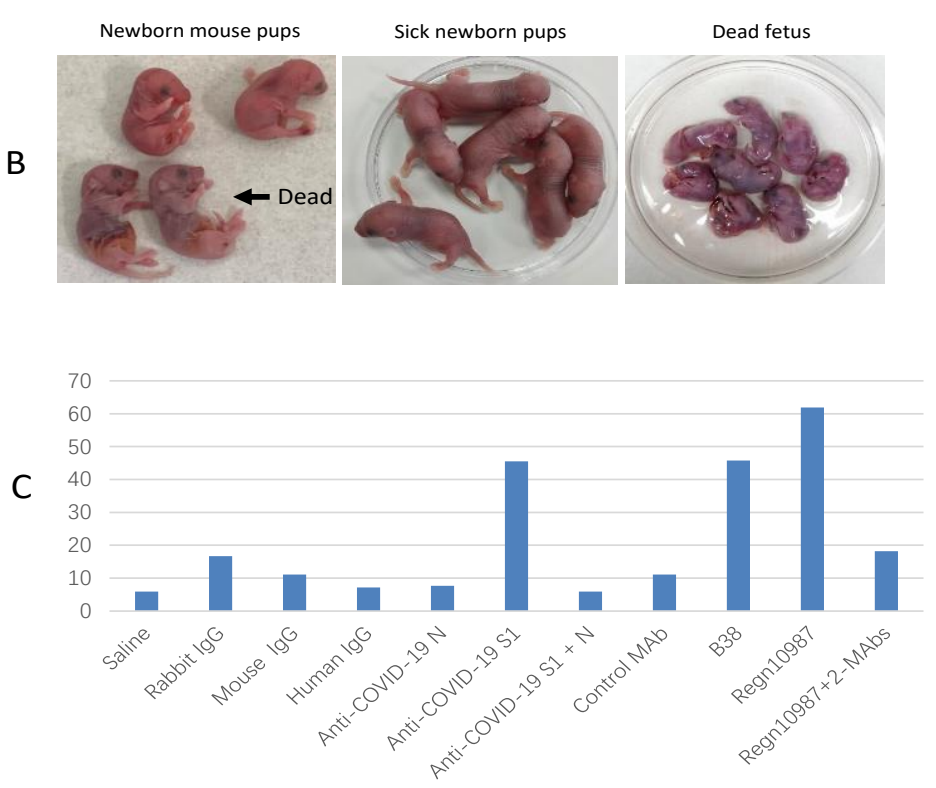

Figure 2. A timed-pregnant mouse model. A: the procedure of injection of anti-coronavirus antibodies into pregnant mice. $\mathrm{B}$ : representative images of mouse pups born to the dames with antibody injection. $\mathrm{C}$ : the rates of sickness and death of newborn mouse pups caused by anti-coronavirus antibodies, and the therapeutic effect of the antibody mixtures of anti-S1 plus anti-N, or REGN10987 plus CR3022-b6 and CC12.3 (2-MAbs). 
Anti-COVID-19 spike antibodies alone induced systemic inflammation and injury in vivo

For further confirmation, a timed-pregnant mouse model without viral infection was used since the surface glycoprotein of fetal organs is not completely developed or unmatured. The purified and endotoxin-free IgG of the anti-COVID-19 S1, anti-COVID-19 N, anti-SARS $\mathrm{S}$, anti-SARS $\mathrm{N}$, human monoclonal antibody of REGN10987 and B38 (Table 1) were used in the mouse model. The purified and endotoxin-free $\mathrm{IgG}$ of healthy sera from rabbit, mouse, and human, as well as the monoclonal antibody of CR3022-b6 were used as controls. Two dosages of each antibody $\operatorname{IgG}$ were injected intraperitoneally $(I P)$ into timed-pregnant mice twice every three days at pregnancy (embryonic) days E15 and E18, respectively, as described in the methods (Figure 2A).

The frequencies of sickness and death of the fetus and newborn mouse pups are summarized in Table 2 and Figure 2C. Injection of REGN10987 into pregnant mice induced significant fetal death and neonatal death of the mouse pups delivered to those dames (Table 2). The fetal death was confirmed by autopsy (Figure 2B). The results with this animal model indicated that
REGN10987 has the highest potential for inducing sickness and death (61.9\%), followed by B38 (45.8\%) and the polyclonal anti-COVID-19 S1 (45.5\%). The polyclonal anti-SARS S also caused significant sickness and death in the newborn mouse pups (37.6\%). In addition, hyperemia at the end of left up and down limbs and a small hemangioma at the side of left eye of one pup was observed. The pup was born to a dame injected with the polyclonal anti-COVID-19 S1 antibody. Neither the healthy control antibodies nor the anti-COVID-19 $\mathrm{N}$ nor the anti-SARS $\mathrm{N}$ caused significant sickness or death of the newborn mouse pups (Table 2).

It was surprising that when the pathogenic polyclonal anti-COVID-19 S1 antibody was mixed with an equal amount of the non-pathogenic anti-COVID-19 N (50 $\mu \mathrm{g}$ $+50 \mu \mathrm{g})$, the sickness and death rate induced by the antibody mixture was significantly lower than that induced by the anti-COVID-19 S1 alone (Table 2). Moreover, the sickness and death rate induced by the highly pathogenic REGN10987 antibody also decreased significantly when a mixture of the antibody and other two non-pathogenic antibodies, CR3022-b6 and

Table 2. The sick and death rates of mouse newborns born to the dames with the injection of anti-coronavirus antibodies

\begin{tabular}{|c|c|c|c|c|c|c|c|}
\hline Injected IgG of & $\mathrm{N}$ & Sick (\%) & Death (\%) & Sick+Death (\%) & Odds Ratio & $95 \% \mathrm{Cl}$ & $P$ value \\
\hline Saline & 17 & 0 & 5.88 & 5.88 & NA & NA & NA \\
\hline Healthy rabbit serum & 6 & 0 & 16.7 & 16.7 & 3.20 & $0.17-61$ & 1.00 \\
\hline Healthy mouse serum & 7 & 0 & 11.1 & 11.1 & 2.67 & $0.14-50$ & 1.00 \\
\hline Healthy human serum pool* & 14 & 0 & 7.14 & 7.14 & 1.23 & $0.07-22$ & 1.00 \\
\hline Anti-COVID-19 N & 13 & 7.69 & 0 & 7.69 & 1.33 & $0.08-24$ & 1.00 \\
\hline Anti-COVID-19 S1 & 22 & 27.3 & 18.2 & 45.5 & 13.3 & $1.5-119$ & 0.01 \\
\hline Anti-COVID-19 S1 + N & 17 & 5.88 & 0 & 5.88 & 0.07 & $0.01-0.7$ & 0.01 \\
\hline Anti-SARS N & 15 & 6.67 & 13.3 & 20.0 & 4.00 & $0.37-43$ & 0.30 \\
\hline Anti-SARS S & 14 & 31.3 & 6.25 & 37.6 & 12.0 & $1.2-117$ & 0.03 \\
\hline MAb-CR3022-b6 & 9 & 0 & 11.1 & 11.1 & 2.00 & $0.11-36$ & 1.00 \\
\hline MAb-B38 & 24 & 33.3 & 12.5 & 45.8 & 13.5 & $1.5-119$ & 0.01 \\
\hline MAb-REGN10987 & 35 & 17.1 & 44.8 & 61.9 & 21.3 & $2.5-179$ & 0.006 \\
\hline REGN10987+2MAbs** & 11 & 0 & 18.2 & 18.2 & 0.17 & $0.03-0.9$ & 0.04 \\
\hline
\end{tabular}

*: Normal IgG pool of 4 healthy individuals without infection or vaccination of coronavirus

** MAb-CR3022-b6 and MAb-CC12.3. Fisher Exact Probability Test, two tailed 
CC12.3, were injected (Table 2). The mixture consisted of $40 \mu \mathrm{g}$ of REGN10987, $20 \mu \mathrm{g}$ of CR3022-b6, and 20 $\mu \mathrm{g}$ of CC12.3. The data suggested that co-existing of non-pathogenic antibodies can reduce the pathogenicity of pathogenic antibodies.

Histology changes. The tissue sections of lungs, brains, hearts, kidneys, intestines, and livers from the newborn mouse pups were stained with hematoxylin-eosin (HE) for histology evaluation. The human IgG or rabbit IgG bound on the tissues in vivo was detected by an immunofluorescent staining with fluorescent labeled anti-human $\mathrm{IgG}$, or anti-rabbit $\mathrm{IgG}$ as secondary antibodies.

Lung inflammation and injury. Acute lung inflammation was observed with the HE stained tissue sections from the mouse pups born to the dames injected with anti-COVID-19 S1, anti-SARS S, REGN10987, and B38 (Figure 3). The lung lesion included pulmonary congestion, alveolar epithelial hyperplasia and thickening, hemorrhage, alveolar atresia, alveolar dilatation, and alveolar fusion (Figure 3 and Figure S1). Infiltration of inflammatory cells at the local lesion areas were also observed. There were insignificant or minor histological changes with the lungs from the pups born to the dames injected with the antibodies of anti-COVID-19 N, anti-SARS N, CR3022-b6, and the control IgGs of human, rabbit, and mouse (Figure 3).

Other organ inflammation and injury. Inflammatory reactions and hemorrhage were also observed with the tissues of kidneys, brains, and hearts from the mouse pups, as mentioned above. The histology of the kidneys from the mouse pups delivered to the dames with the injection of anti-COVID-19 S1, anti-SARS S, B38, and REGN10987 showed acute tubular injury. Renal tubular epithelial cells showed granular or vacuolar degeneration, dilated or obstructed lumen, renal interstitial edema with a small amount of inflammatory cells infiltration, and some of the epithelial cells fell off (Figures 3 and S2). The kidney injury caused by REGN10987 was the most significant (Figure 3). Furthermore, small amounts of cerebral hemorrhage or inflammatory cell infiltration was observed in the brains of mouse pups delivered to a dame injected with antibodies of anti-COVID-19 S1, anti-SARS S, B38, and REGN10987 (Figure 3 and S2). Additionally, myocardial hemorrhage was observed in the hearts of

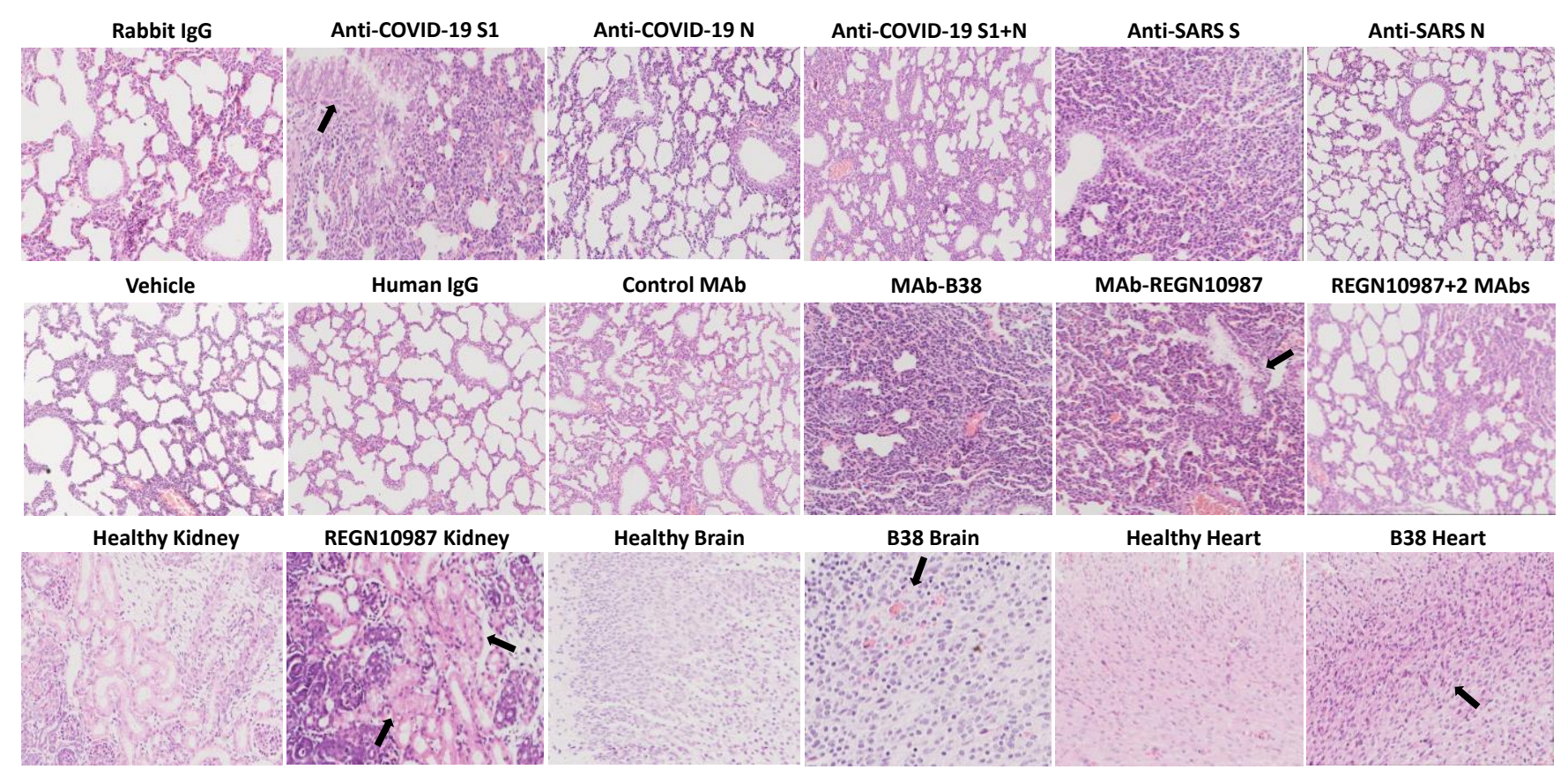

Figure 3. The representative images of the histological changes of lungs (top 2 rows), kidneys, brains, and hearts (bottom row) from the newborn mouse pups delivered to the dames injected with anti-coronavirus antibodies, and control antibodies of human IgG, rabbit IgG and human monoclonal antibody (MAb) of CR3022-b6 (Control MAb); or the dames treated with antibody mixtures. 2MAbs: human monoclonal antibodies of CR3022-b6 and CC12.3. 
the mouse pups delivered to the dames injected with anti-COVID-19 S1, anti-SARS S, and B38 (Figure 3 and S2). Lastly, myocardial swelling and inflammatory cell infiltration were observed in a mouse pup delivered to a dame injected with antibodies of B38 (Figure 3). There were insignificant or minor histological changes with the tissues from the pups born to the dames injected with the antibodies of anti-COVID-19 N, anti-SARS N, CR3022-b6, and the control IgGs of human, rabbit, and mouse.

Taken together, the in vivo results of the virus-free animal model proved that certain antibodies specific to the spike proteins of the coronaviruses such as COVID-19 and SARS-CoV viruses can induce, through the mechanism of Antibody Dependent Auto-Attack (ADAA), significant fetal and neonatal deaths and the systemic inflammation or injury of lung and other organs in vivo. The results are consistent with the clinical observations of COVID-19 patients with severe illnesses ${ }^{5}$. Therefore, certain antibodies of anti-COVID-19 S1, anti-SARS-CoV S, B38, and REGN10987 are pathogenic and induce ADAA. The pathogenic antibodies and ADAA are probably responsible for the serious conditions of a severe COVID-19 infection. On the other hand, the antibodies of anti-COVID-19 N, anti-SARS-CoV N, and CR3022-b6 are non-pathogenic since they did not induce significant adverse reactions in vivo.

\section{Inflammatory cytokines}

As further evidence of the pathogenicity of the antispike antibodies, the cytokine levels of monocyte chemotactic protein 1 (MCP-1), tumor necrosis factor- $\alpha$ (TNF- $\alpha$ ), interleukin-4 (IL-4), IL-6, and IL-10 in the sera of mouse pups were tested by a multiplex Luminex assay kit (Millipore) according to manufacturer's instructions. The results of MCP-1 are summarized in Figure 4.

Both the pathogenic anti-COVID-19 S1 and REGN10987 induced significantly higher levels of MCP-1 (Figure 4). Consistent with the surprising results of the histological changes, the treatment using the antibody mixture comprised of the pathogenic anti-COVID-19 S1 and the non-pathogenic anti-COVID-19 N, significantly reduced the cytokine levels of MCP-1 ( $\mathrm{P}<0.001)$ compared to that induced by the anti-COVID-19 S1 alone (Figure 4). In addition, the treatment with the antibody mixture comprised of the pathogenic REGN10987 and two non-pathogenic antibodies of CR3022-b6 and CC12.3 also significantly reduced the level of MCP-1 ( $<$ < 0.001) compared to that induced by REGN10987 alone (Figure 4). The levels of other cytokines were not significantly elevated, probably due to the undeveloped immunity of the newborn mouse pups. The results were consistent with the results of the sickness and death rates (Table 2) and the histology changes (Figure 3). The data demonstrated that 1) pathogenic antibodies alone can induce high levels of inflammatory cytokines and have the potential to induce a cytokine storm or cytokine release syndrome (CRS); and 2) co-existence of non-pathogenic antibodies can reduce the inflammatory cytokine release induced by pathogenic antibodies and prevent the possible cytokine storm or CRS caused by the pathogenic antibodies.

\section{In vivo antibody binding to multiple organs of mouse pups}

As more evidence of the pathogenic ADAA of the anti-COVID-19 spike antibodies, in vivo antibody binding to tissues of mouse pups was detected using an immunofluorescent staining as described in methods.
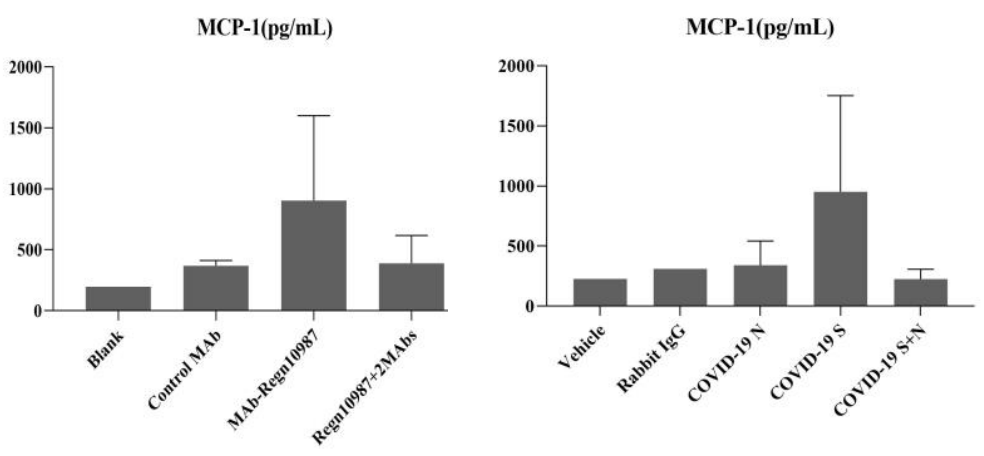

Figure 4. The cytokine levels of MCP-1 in mouse sera from the newborn mouse pups born to the dames with antibody injection alone of the anti-coronavirus antibodies or the dames treated with antibody mixtures. 
The human and rabbit anti-COVID-19 spike antibodies were significantly detectable at the inflammatory and lesion areas of the tissues of lungs, kidneys, brains, and provided the in vivo evidence for the pathogenic ADAA of some anti-spike antibodies of COVID-19 virus and SARS-CoV virus.

$$
\text { A }
$$

$\frac{\mathrm{O}}{\mathrm{O}}$
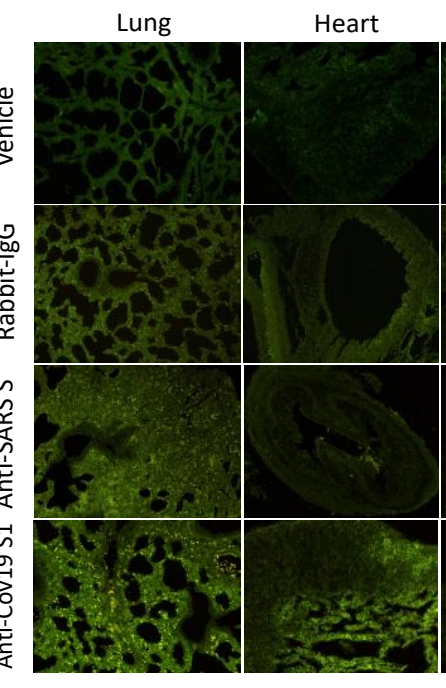

Kidney
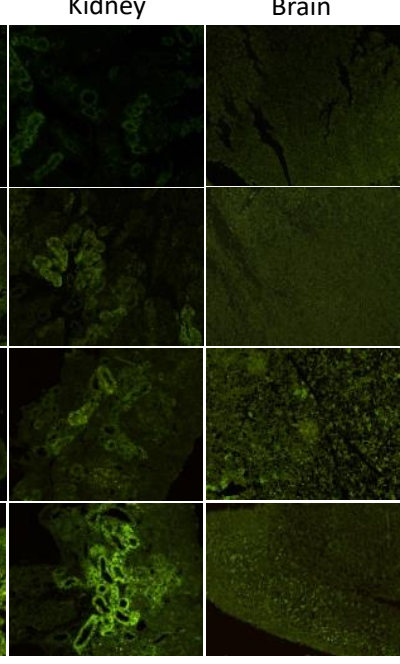

B

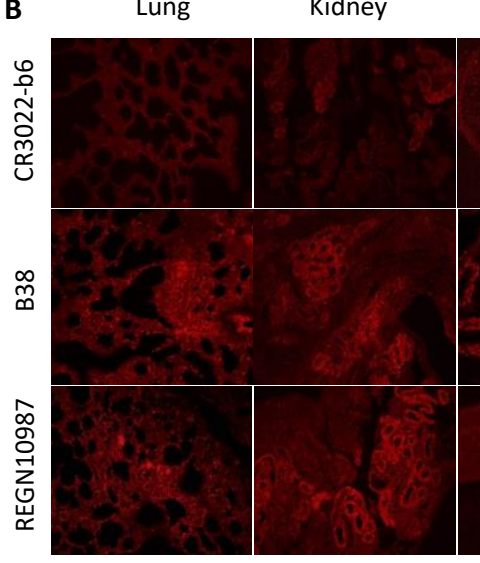

Heart

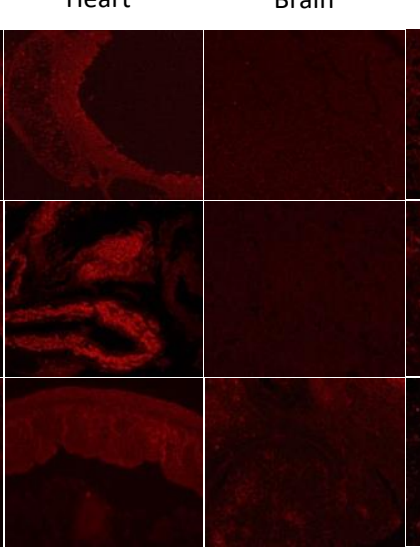

Liver

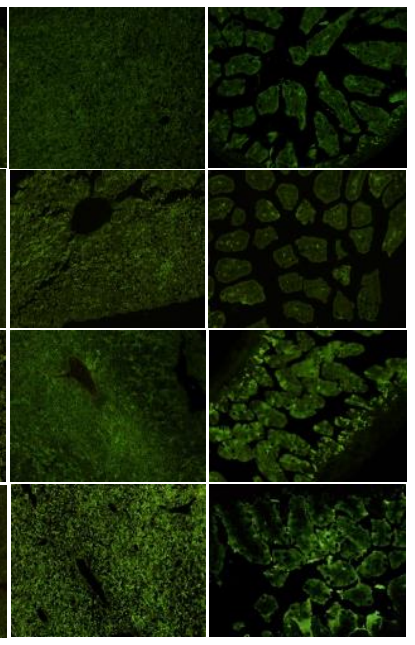

Liver

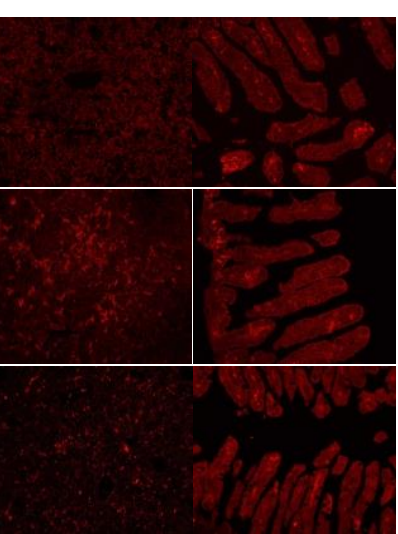

Figure 5. Detection of anti-coronavirus antibodies bound in vivo to the lesion areas of multiple organs of the mouse pups born to the dames with antibody injection at E15 and E18. hearts, livers, and intestines from the mouse pups with severe sickness (Figure 5). Those mouse pups were delivered to the dames injected with the pathogenic antibodies of anti-COVID-19 S1, anti-SARS-CoV S, REGN10987, and B38 (Figure 5). Neither human IgG nor rabbit IgG was significantly detected on the tissues of the pups treated with the non-pathogenic antibodies of anti-COVID-19 N, anti-SARS N, and CR3022-b6 (Figure 5). The results indicated that certain anti-COVID-19 spike antibodies went through the placenta, bound to the fetal tissues, activated the self-attack immune responses, and led to the systematic inflammation and injuries of multiple organs such as the lungs, kidneys, heart, and brain. The results are consistent with those of histological changes (Figure3)

\section{Binding of pathogenic antibodies to fetal and diseased tissues}

In order to further evaluate the pathogenicity of the pathogenic anti-COVID-19 spike antibodies in humans, the REGN10987 with the highest pathogenic potential was tested for antibody binding to various human fetal tissues, or mutiple human diseased tissues from tissue array slides (US Biomax). The results are shown in Figure 6. Antibody REGN10987 bound to mutiple human fetal tissues of the lungs, heart, kidneys, brain, pancreas, liver, thymus, and testicles, but not of the esophagus (Figure 6A). In addition, the REGN10987 also bound to the fetal tissues of retina and coroid, sclera, and eye ball (data not shown). The data indicate that the unmatured fetal tissues are vulnerable to a 
pathogenic antibody such as REGN10987. In addition, REGN10987 bound broadly to the human inflammatory tissues or cancer tissues of the respiratory, cardiovascular, urinary, and digestive systems (Figure 6B-6C). The inflammatory tissues were from patients suffering from pneumonia, bronchitis, bronchiectasis, valvular disease, rheumatoid valvular disease, myocarditis, esophagitis, gastritis, collitis, appendicitis, pancreatitis, and hepatitis. The cancer tissues were from patients with small cell lung carcinoma, kidney clear cell carcinoma, and myxoma. The results indicate that most of the actively proliferating cells or tissues such as inflammatory tissues or cancer tissues are vulnerable to a pathogenic anti-COVID-19 S1 antibody such as

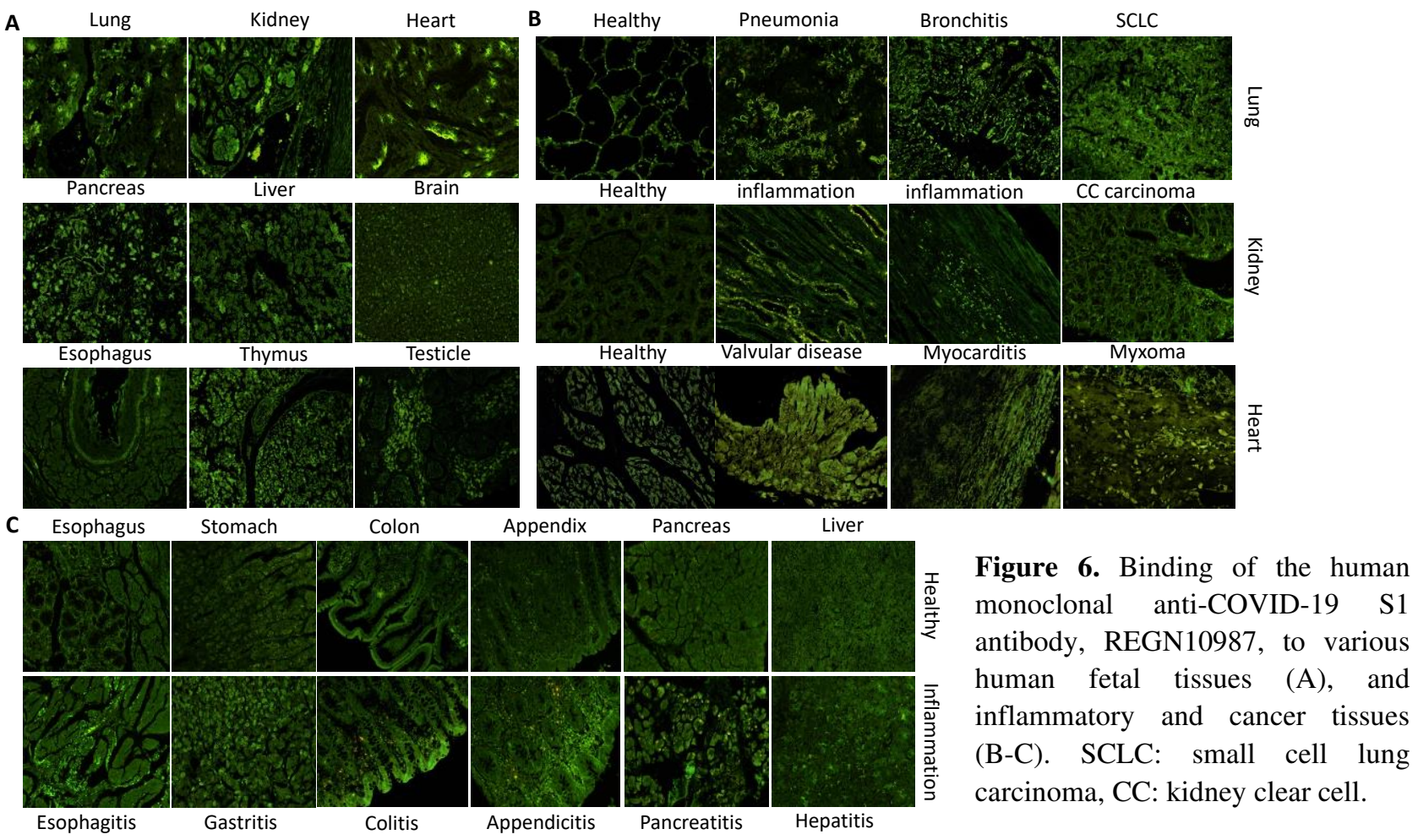

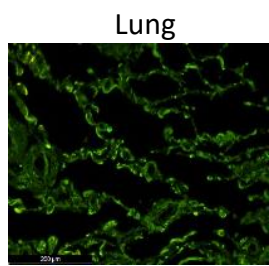

Pancreas

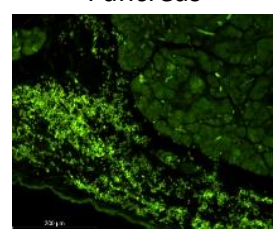

Adenohypophysis

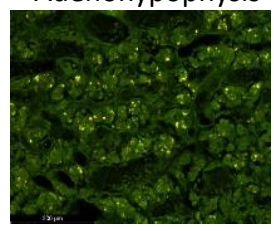

Kidney

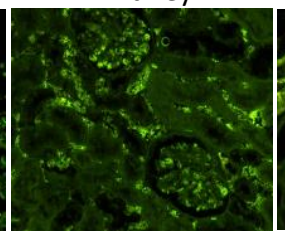

Adrenal gland

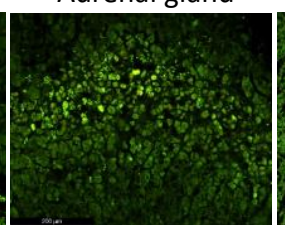

Testis

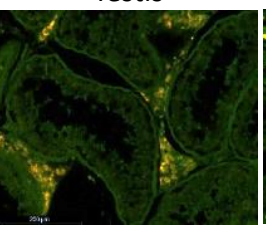

Kidney

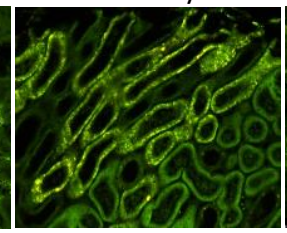

Peripheral nerve

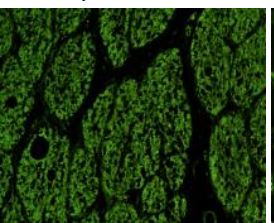

Prostate

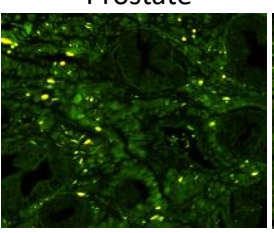

Stomach

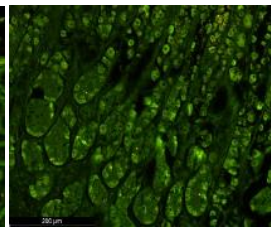

Thyroid gland

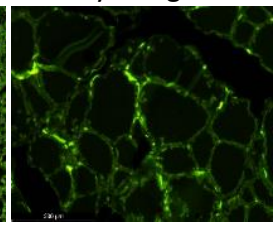

Uterine cervix

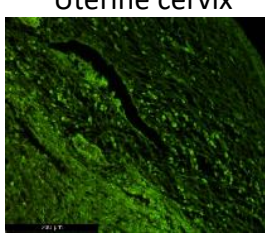

Small intestine

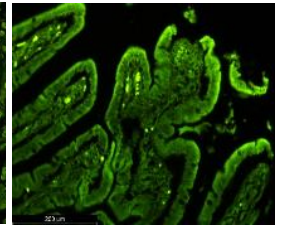

Spleen

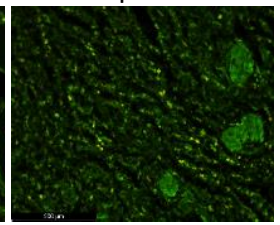

Bone marrow

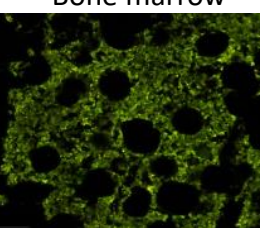

Figure 7. Binding of the REGN10987 to various normal human tissues. 


\section{REGN10987.}

\section{Binding of pathogenic antibodies to human healthy} tissues

As more evidence of the pathogenicity of the pathogenic anti-COVID-19 S1 antibodies, REGN10987 and mutiple healthy human tissues from a tissue array slide (US Biomax, FDA999w) were used to further evaluate the pathogenicity of REGN10987. The tissue array slide was comprised of 32 types of normal human organs encompassing most of human tissues, representing FDA guidelines for antibody cross-reactivity testing. The results showed that REGN10987 bound significantly to human healthy tissues of lung, kidney, pancreas, stomach, intestine, adrenal gland, peripheral nerve, thyroid gland, spleen, adenohypophysis, testicle, prostate, bone marrow, uterine cervix of cancer adjancent normal tissue (Figure 7). In addition, REGN10987 also bound to the tissues of parathyroid gland, pericardial mesothelium, and adjacent normal sclera of eye (data not shown). The data indicate that certain anti-COVID-19 S1 antibodies, such as REGN10987, are highly pathogenic because it has the high potential to bind to healthy human tissues, activating self-attacking immune responses (ADAA) and inducing serious adverse reactions in vivo. Based on the results, clinical detection of pathogenic antibodies during the COVID-19 infection may be helpful in predicting the consequences of a patient with a serious infection.

Taken together, the in vitro and in vivo data of the current study revealed that certain pathogenic antibodies specific to the COVID-19 spike protein can be the cause of a serious COVID-19 infection, and can cause serious complications during COVID-19 infections through ADAA. Further, the pathogenic antibodies can bind to unmatured fetal cells or tissues and cause abortions, postpartum labors, still births, and neonatal deaths of pregnant females.

It should be noted that the majority ( $70 \%$ or more) of the anti-COVID-19 spike antibodies inducible by the COVID-19 virus are non-pathogenic since the pathogenic antibodies consist of less than $30 \%$ of the total number of antibodies, according to the data of monoclonal antibodies in this study.

\section{Discussion}

The current study revealed the pathogenic roles and the new mechanism of action (ADAA) of certain antibodies specific to the spike proteins of coronaviruses such as the COVID-19 virus and the SARS-CoV virus (Figure 8). We had discovered that in a mouse model, pre-injection of anti-influenza immune sera induced

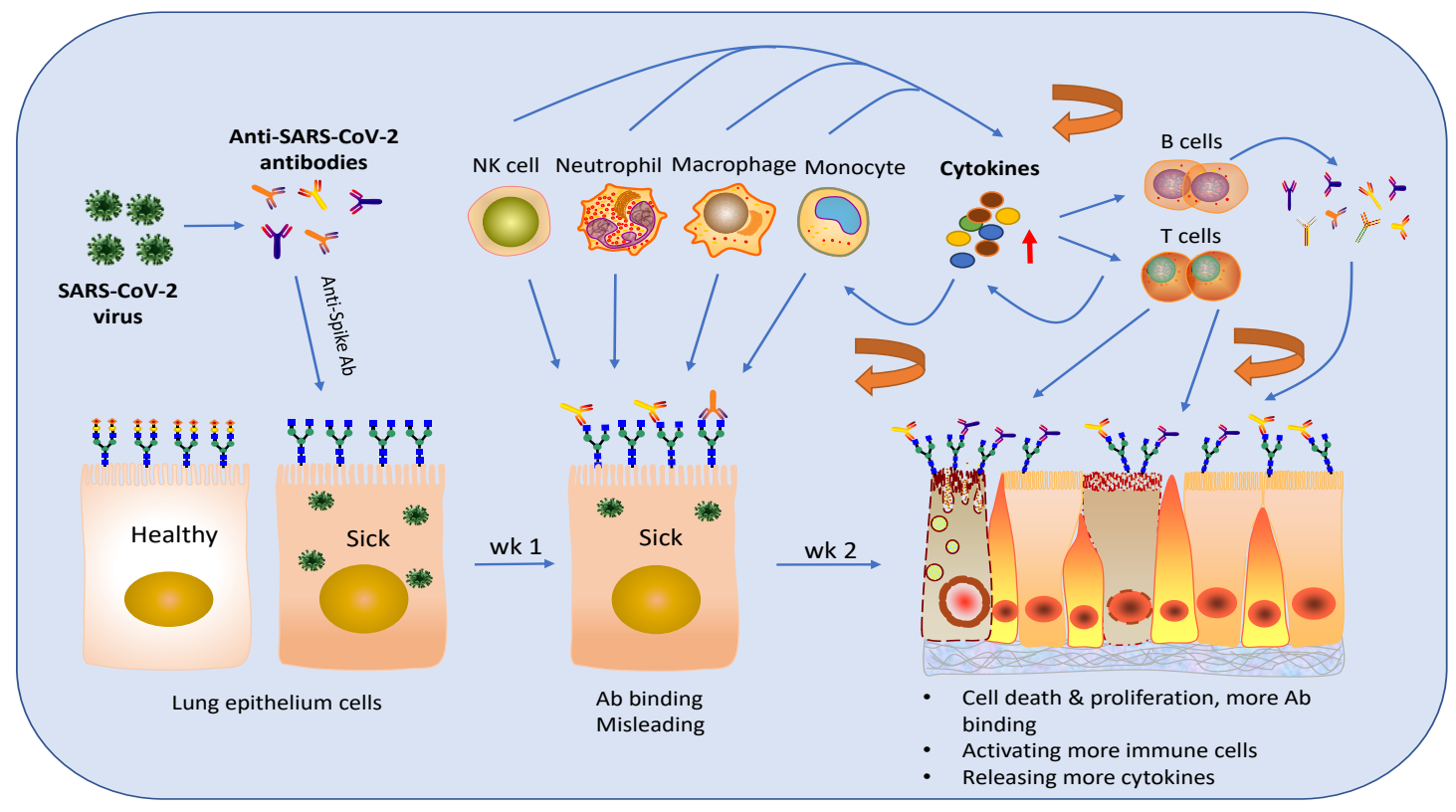

Figure 8. Antibody Dependent Auto-Attack (ADAA) of pathogenic anti-spike antibodies of COVID-19 virus. 
more severe infections than the mice infected with an influenza virus alone ${ }^{14}$. Wang and co-workers reported that anti-SARS-CoV spike antisera promoted SARS infection through antibody-dependent enhancement (ADE) in vitro ${ }^{22}$. Liu and co-workers reported that anti-SARS-CoV spike immune sera induced by a SARS-CoV vaccine caused acute lung injury by promoting MCP1 and IL-8 production and monocyte or macrophage recruitment and accumulation in SARS-CoV infected macaque models ${ }^{23}$. The previously reported mechanism of action (MOA) of these anti-spike antibodies is ADE-based, in that the antibodies enhance viral infectivity ${ }^{24}$. The current study, revealed for the first time, the self-cell targeting MOA of pathogenic antibodies, in which the antibodies bind to host vulnerable cells or tissues and mislead immune responses to attack host-self (ADAA). Our study also explored a new mechanism of pathogenisis (MOP) of highly pathogenic viral infections. The MOP is caused by the pathogenic antibodies inducible by highly pathogenic viruses such as the COVID-19 virus and SARS-CoV virus. Moreover, the pathogenic antibodies may be related to the cause of infection-related autoimmune diseases, including those in COVID-19 long haulers, through ADAA.

\section{Dualistic roles of anti-viral antibodies}

Based on the traditional concept, the antibodies induced by an infectious pathogen are protective to a host because they can neutralize the pathogen and prevent or treat the infectious disease. Nevertheless, the roles of such antibodies can be dualistic. Not wishing to be bound to theory, we noticed that some antibodies can cross react to host cells or tissues and trigger immune reactions to attack the self-cells and self-tissues (ADAA). The data of the current study showed such pathogenic actions of anti-spike antibodies of the COVID-19 and SARS viruses.

Sialic acids are predominant components of the mucous membrane at the outer surface of cell membranes and mainly act as biological masks or receptors ${ }^{25}$. Cells or tissues with sialic acid are recognized as "self" ${ }^{25}$. After the loss of sialic acids the cellular structures become "non-self" 25 , which can activate immune responses. Sialic acids are also an important attachment molecule of receptors for some viruses, such as coronaviruses and influenza viruses ${ }^{25,21}$. During an infection of such viruses, the sialic acid on the infected cells such as lung epithelium cells could be removed or destroyed by the receptor destroying enzyme (RDE) of a coronavirus such as the COVID-19 virus, or the sialidase of influenza viruses. The damaged cells with missing sialic acid on the cell surface become vulnerable to the pathogenic antibodies induced by the virus (Figure 8). For example, the current study showed that antibodies specific to the SARS-CoV-2 spike and the SARS-CoV spike glycoprotein could significantly bind to the damaged lung epithelium A549 cells (Figure 1) and kidney embryonic HEK-293 cells with missing sialic acid on the cell surface. The antibody binding could activate and mislead the immune response to attack self and induce the injury of multiple organs in vivo. For example, injection of the antibodies specific to the COVID-19 S1 or the SARS-CoV S proteins to pregnant mice induced fetal and neonatal deaths and the injury of multiple organs of mouse pups born to the dames, as shown in Figures 1-4 and Table 2. In contrast, the non-pathogenic antibodies of anti-COVID-19 $\mathrm{N}$ and anti-SARS $\mathrm{N}$ did not induce significant injury in vivo. Neither the anti-COVID-19 $\mathrm{N}$ nor the anti-SARS $\mathrm{N}$ antibodies significantly bound to the healthy A549 cells nor to the damaged A549 cells (Figure 1E). The fetal model was selected because many fetal tissues, including the surface glycoproteins, are unmatured and vulnerable to the pathogenic antibodies.

Seven naturally occurring human monoclonal antibodies specific to the COVID-19 S1 (S-RBD) protein $^{15,16,17,18,19,20}$ were analyzed for their pathogenic potential, with the in vitro assay as described above. The results showed that all antibodies except one $(85.7 \%)$ did not bind to the healthy A549 cells. Neverthless, two (28.6\%) antibodies of B38 and REGN10987 significantly bound to the damaged A549 cells (Figure 1C) and were selected as potential pathogenic antibodies for further confirmation by the in vivo animal model. The results of the virus-free animal experiments showed that the two antibodies alone are highly pathogenic and induced significant fetal and neonatal deaths and the injury of multiple organs of mouse pups born to the dames, as shown in Figures 1-4 and Table 2. In contrast, another antibody of the seven monoclonal anti-COVID-19 S1 antibodies, CR3022-b6, served as a control antibody and did not induce significant injury in 
vivo (Figures 1-4 and Table 2). The data indicated that the in vitro assay is useful for rapid screening of potential pathogenic antibodies, and the virus-free animal model is helpful for confirmation of the pathogenicity of these particular antibodies. It should be noted that the effect of a pathogenic antibody on the mouse fetus could be reduced if the antibody binds to the mother's tissues.

A pathogenic antibody can be induced during a highly pathogenic infection, such as the COVID-19 infection. The discovery of pathogenic antibodies and their ADAA mechanism may solve the mystery of the possible mechanisms of pathogenesis (MOP) of serious infectious diseases, serious complications, and sequela of a viral infection, particularly of a highly pathogenic viral infection such as the COVID-19 infection. This may also explain the cause of cytokine storms and cytokine release syndrome (CRS), and infection-related autoimmune diseases (including those suffered by COVID-19 long haulers ${ }^{26}$ ), infection- relating cancers, and other possible disorders inducible by pathogenic antibodies. The diseases or conditions caused by pathogenic antibodies further include abortion, postpartum labor, still birth and neonatal death of pregnant females related to an infection.

\section{A new pathogenic mechanism of viral infections}

The in vitro and in vivo data of the current study support a new MOP of highly pathogenic viruses such as the COVID-19 virus. The MOP includes: 1) a highly pathogenic respiratory virus such as the COVID-19 virus causes the initial, primary injury such as local inflammation and cellular damage with missing sialic acids of its target organ, such as lungs, typically within week one of the infection; 2) anti-viral antibodies, including pathogenic antibodies, elevate from week one. The pathogenic antibodies bind to the damaged or the inflammatory cells of the target organ (e.g., lungs) (Figure 8) as well as to other organs with similar injuries (e.g. kidney, brain and heart); 3) the antibody binding activates and misleads the immune response to attack the self cells or tissues (ADAA), and induces further damage (the secondary injury); 4) the secondary injury persistently adds further damage to the primary injury, creating a snowball effect, and causes serious conditions such as ARDS, cytokine storms, and even death as the antibodies reach peak levels from week one to weeks 2-3 (Figure 8); and 5) the self-attacking immune responses (ADAA) misled by the pathogenic antibodies can be persistent, and can accumulate after viral clearance and cause autoimmune diseases as long as the antibodies continue to exist.

The primary injury is limited, short, and decreases as the virus is cleared, as seen in regular influenza infections. That means the virus itself is not sufficient to cause serious conditions such as ARDS, cytokine storms, and death. On the other hand, the secondary injury caused by the pathogenic antibodies is longer, broader, and additive because antibodies persist much longer than viruses and can bind nonspecifically to other inflammatory tissues or organs besides lungs. Thus, the superposition of the ADAA reactions caused by pathogenic antibodies can lead to serious conditions such as ARDS, cytokine storm, and death.

The new MOP can explain why the majority of patients with serious respiratory viral infections such as a COVID-19 infection or a highly pathogenic influenza infection died after week one, especially at weeks 2-3 of the infection course ${ }^{3,27}$, as that matches the time period of antibody peak levels. The new MOP can also explain why the majority of severe or lethal infections of the 1918 influenza pandemic happened to the young ${ }^{27}$, because younger people could induce higher levels of anti-viral antibodies, including pathogenic antibodies. Similarly, certain pathogenic antibodies inducible by other infectious pathogens, such as influenza viruses may also cause serious adverse reactions or autoimmune diseases through this MOP (study ongoing).

\section{Cells or tissues vulnerable to pathogenic antibodies}

As shown in Figure 6, the highly pathogenic REGN10987 antibody bound to the majority of human fetal tissues and inflamatory tissues, as well as certain cancer tissues. A common feature of those tissues, including the cancer tissues, is that they consist of actively proliferating cells. Therefore, those cells and inflamatory tissues are vulnerable to pathogenic antibodies, and their interaction with the pathogenic antibodies (ADAA) can be the cause of 1) serious infections, particularly highly pathogenic viral infections such as the COVID-19 infection; 2) serious complications of infections such as ARDS, cytokine storm, or cytokine release syndrome (CRS); and 3) 
infection-relating inflammation such as pneumonia and kidney failure (Figures 1-4). Further, unmatured fetal cells or tissues are vulnerable to pathogenic antibodies (Figure 6A). Thus, pathogenic antibodies, through ADAA, may be the cause of abortions, postpartum labors, still births and neonatal deaths of pregnant females (Figure 1B and 1C).

\section{Pathogenic antibodies and autoimmune diseases}

Many autoimmune diseases are related to viral infections $^{28}$, yet the pathogenic mechanisms have remained unclear so far. The current study discloses, for the first time, that most inflammatory tissues are vulnerable to pathogenic antibodies and ADAA (Figure 6B). Chronic inflammation is a feature of most autoimmune diseases. When an infection occurrs, antibodies including pathogenic antibodies are induced and last from months to half a year. The pathogenic antibodies can readily bind to the pre-existing vulnerable inflammatory tissues of an autoimmune disease, and mislead immune responses to attack the body's own tissues. This ADAA process can be repetitive and additive as antibodies elevate and induces significant reactions. The occurrence time and pathogenic course of an autoimmune disease caused by pathogenic antibodies and ADAA can be short or long, depending on the amount of vulnerable cells or tissues and the amount of pathogenic antibodies. The pathogenic antibodies generally persist for months to half a year, and thus the autoimmune disease can occur during that period, especially during the period of antibody peak levels.

COVID-19 long haulers have been reported and the causes remain a mystery ${ }^{26}$. The current study provides a possible pathogenic mechanism of COVID-19 long haulers. Despite the virus being cleared in the recovered COVID-19 patients, the anti-viral antibodies remained and could exist for months to half a year or longer. Certain pathogenic antibodies could bind to vulnerable cells or tissues, such as inflammatory cells induced during the COVID-19 infection, and cause persistent adverse reactions such as chronic inflammation of multiple organs. Thus, with the mechanism of ADAA, pathogenic antibodies can also be responsible for the longer term effects of the COVID-19 infection. For example, a highly pathogenic anti-spike antibody such as REGN10987 can bind to peripheral nerves and may cause abnormalities of muscles, vision and taste. Similarly, the pathogenic antibodies inducible by other infectious pathogens such as an avian influenza virus can be responsible for the adverse reactions or autoimmune diseases caused by the infections such as Guillain-Barré syndrome (GBS). Additionally, in a chronic viral infection (e.g., an HIV infection), pathogenic antibodies can repeatedly stimulate cellular proliferation for a long time and an infection-related cancer can occur if the cellular proliferation loses control.

\section{Better vaccines}

It was surprising that when the pathogenic anti-COVID-19 S1 antibodies was mixed with an equal amount of the non-pathogenic anti-COVID-19 N antibodies, the sickness and death rates caused by the antibody mixture was significantly decreased compared to the results of the injected anti-COVID-19 S1 antibody alone (Table 2 and Figure 1C). A similar result was observed with the highly pathogenic REGN10987 as well with an antibody mixture of the REGN10987 and the two non-pathogenic monoclonal antibodies of CR3022-b6 and CC12.3 (Table 2 and Figure 1C). The data suggested that co-existence of non-pathogenic antibodies can reduce the ADAA of pathogenic antibodies. Thus, a vaccine capable of inducing multivalent antibodies may be safer, in which at least one kind of antibody is of the non-pathogenic kind that induces fewer adverse reactions. One example of such a vaccine is the traditional inactivated viral vaccine (e.g. inactivated COVID-19 vaccine) which induces multivalent antibodies specific for multiple antigens of a virus. As another example, a recombinant or mRNA COVID-19 vaccine capable of inducing the antibodies specific to not only the spike protein but also to the nucleocapsid proteins, or a non-spike protein of the SARS-CoV-2 virus may be safer (patent pending). Despite the mechanism of action being unclear, it is not likely that the non-pathogenic antibodies affected the ADA actions of the pathogenic antibodies through competitive binding since neither the anti-COVID-19 S1 nor the anti-COVID-19 N bind to the same antigens. Neither the REGN10987 nor the other two non-pathogenic antibodies bind to the same epitope of the S1 protein of the COVID-19 virus. We hypothesize that non-pathogenic antibodies affect the ADAA of 
pathogenic antibodies through diluting and space obstructing or interrupting the attachment of pathogenic antibodies to vulnerable cells or tissues.

\section{Novel clinic interventions based on the new} mechanism of pathogenesis

Based on the new MOP of the highly pathogenic viral infection inducible by pathogenic antibodies, novel clinic interventions for treating and preventing a serious condition of the COVID-19 infection may be developed through interrupting the binding of the pathogenic antibodies to host vulnerable cells, tissues, and organs. For example, the following products and approaches may be effective for the treatment of a serious COVID-19 infection: 1) for patients with serious conditions, therapies capable of removing the pathogenic antibodies, such as replacing the patient's plasma with uninfected healthy human plasma, may be effective and should be performed as soon as possible; 2) immunoglobulin products, serum or plasma from not only recovered patients but also from healthy individuals may be helpful for symptom relief through diluting and interrupting the binding of pathogenic antibodies; and 3) pathogen-derived products such as viral antigens or antigen fragments, and synthetic peptides may be effective (neutralizing pathogenic antibodies) (patent pending).

For the diagnosis of a severe COVID-19 infection, determination of the levels of anti-COVID-19 spike antibodies may be important and helpful to predict the condition of a patient with a serious infection. This is because the virus can become undetectable after one week, but the antibodies induced by the virus can elevate from week one and reach peak levels at weeks 2-3, accompanied by the development of serious conditions such as ARDS, cytokine storms, and death. Higher levels of the anti-COVID-19 spike antibodies may indicate a worsening of the infection.

In summary, our study revealed the roles of "pathogenic antibodies" in viral infections. These pathogenic antibodies act through a novel mechanism of action that we have term "Antibody Dependent Auto-Attack" (ADAA). In particularly, we explored the ADAA of highly pathogenic respiratory viral infections, such as COVID-19. The pathogenic antibodies can be induced during an infection, bind to vulnerable cells or tissues such as actively growing cells, and initiate a persistent self-attack immune response, leading to serious conditions including ARDS, cytokine storms, and death. Further, the pathogenic antibodies, through ADAA, may also be responsible for infection-related autoimmune diseases, including those experienced by COVID-19 long haulers. Novel clinical interventions for diagnosis and treatment, including improved vaccines based on the ADAA of pathogenic antibodies, can be developed. In addition, the new concept and the mechanism of ADAA explored by this study may also be applicable to other infectious diseases, such as highly pathogenic influenza infections (study ongoing).

\section{Methods}

Production of human anti-SARC-CoV-2 spike monoclonal antibodies

Seven naturally occurring human monoclonal antibodies specific to the receptor binding domain (RBD) of the spike protein one (S1) of the SARS-CoV-2 virus have been reported by others ${ }^{15,16,17,18,19,20}$. The seven monoclonal antibodies were reproduced for research use only (HuaAn McAb Biotechnology) according to the gene sequences from the NCBI crystal structure site (Table 1). The features and information of the antibodies are listed in Table 1. Paired heavy chain and light chain plasmids of anti-Spike human antibody were transiently transfected into HEK-293FE cells at a mass ratio of $1: 2$ with polyetherimide (Polysciences). Transfected cells were suspension-cultured in a $5 \% \quad \mathrm{CO}_{2}$ containing atmosphere at $37^{\circ} \mathrm{C}$ and the condition medium (Gibco) was refreshed 8 hours after transfection. After 7 days, the supernatants were centrifuged at $10000 \mathrm{r}$ per min (rpm) and purified with Protein A prepacked column (Senhui Microsphere Technology). Purified antibodies were quantified by Nanodrop or BCA Quantification Kit (Beyotime).

Antibody quality control was performed using an antigen down ELISA. $1 \mu \mathrm{g} / \mathrm{ml}$ antigens of COVID-19 Spike-RBD-mFc (HuaAn McAb Biotechnology) were coated on ELISA plate at $4^{\circ} \mathrm{C}$ for overnight. The plate was blocked with $1 \%$ BSA and coated with anti-spike human antibody as first antibody for 1 hour at $37^{\circ} \mathrm{C}$ successively. Uncombined free antibodies were washed with TBST, the secondary anti-human IgG-HRP 
(Abcam) were added onto the plate, and the plate was incubated at $37^{\circ} \mathrm{C}$ for 30 minutes. Binding affinity of the anti-spike human antibodies were analyzed with the signature of $\mathrm{OD}_{450} \mathrm{~nm}$ absorption.

\section{Anti-COVID19 $N$ protein antibody production}

Two months old New Zealand white rabbits were subcutaneously immunized with $250 \mu \mathrm{g}$ of purified COVID19-N protein ((HuaAn McAb Biotechnology). The rabbits received 3 booster injections at 2-week intervals with $500 \mu \mathrm{g}$ purified COVID19-N protein. Serum titer of anti-COVID19-N antibodies were validated by an antigen down ELISA after each immunization. Rabbit serum were then harvested, centrifuged at 10,000 rpm for 10 minutes and purified with Protein A prepacked column (Senhui Microsphere Technology). Purified antibodies were quantified by Nanodrop or BCA Quantification Kit (Beyotime).

\section{Removal of endotoxin from antibody solutions}

Antibody solutions were treated with $1 \% \mathrm{v} / \mathrm{v}$ Triton $\mathrm{X}-114$ in ice for 10 minutes. The solutions were then incubated at $37^{\circ} \mathrm{C}$ for 5 minutes, whereupon two phases formed. The antibody solutions were centrifuged at $12,000 \mathrm{rpm}$ for 5 minutes at room temperature, and carefully aspirate the upper aqueous phase. Endotoxin-free antibodies were quantified by BCA Quantification Kit (Beyotime).

\section{Other antibodies}

The rabbit polyclonal antibodies specific for the recombinant spike one (S1) proteins of the SARS-CoV-2 virus, the recombinant spike proteins of SARS-CoV virus, and the mouse monoclonal antibody specific for the recombinant nucleocapsid $(\mathrm{N})$ proteins of the SARS-CoV virus were purchased from Bioss Antibodies (Beijing).

\section{Digestion of A549 cells with neuraminidase}

The cells of human lung epithelium cell line A549 were washed once with the digestion buffer for neuraminidase (Roche), the cells were centrifuged at $2000 \mathrm{rpm}$ for 5 minutes, and the supernatant was discarded. The digestion buffer was consisted of $50 \mathrm{mM}$ of sodium acetate, $3 \% \mathrm{BSA}$, and $2 \mathrm{mM}$ of $\mathrm{CaCl}_{2}$ in PBS (pH5.5-6.0). The cells were resuspended with the digestion buffer and divided to multiple tubes, each tube contained about $10^{6}$ of cells in $200 \mu$ of the digestion buffer containing $50 \mu \mathrm{U}$ of neuraminidase (Roche). The tubes were incubated at $37^{\circ} \mathrm{C}$ for one hour. Then the cells were washed with PBS once and proceeded for flow cytometry assay. A549 cells without the treatment of neuraminidase were used as controls.

\section{Flow cytometry}

A549 cell with or without the treatment of neuraminidase were tested for the binding of anti-coronavirus antibodies as mentioned above. Each $2 \times 10^{5}$ of cells in $200 \mu \mathrm{l}$ of $1 \%$ BSA-PBS were incubated in ice with each antibody at a concentration of 0.5 $\mathrm{mg} / \mathrm{ml}$ for one hour, washed once with PBS. The cells were resuspended with $200 \mu \mathrm{l}$ of $1 \% \mathrm{BSA}$-PBS and 0.25 $\mathrm{mg} / \mathrm{ml}$ of either PE-labeled goat anti-human IgG (Abcam) or FITC-labeled goat anti-rabbit IgG (SouthernBiotech), incubated in ice for 30 minutes, and washed once with PBS. The cells were resuspended with $200 \mu \mathrm{l}$ of PBS and detected with a flow cytometer of Accuri 6 (BD Biosciences). The fluorescent labeled wheat germ agglutinin (WGA), which specifically binds to sialic acid (Vector), was used as a control (Figure 1A).

\section{A timed-pregnant mouse model}

A timed-pregnant mouse model without viral infection was developed using the anti-coronavirus antibodies as mentioned above, to evaluate the pathogenic action of anti-coronavirus antibodies. The animal experiments were performed at the Center of Laboratory Animals of Hangzhou Normal University. The protocol for the animal experiment was approved by the Laboratory Animal Welfare and Ethics Committee of Hangzhou Normal University. The CALAS No. is 2020244. The animal experiments were performed three times.

Animal SPF-grade C57BL/6J pregnant mice at pregnancy (embryonic) day E13-E14 were purchased from Shanghai SLAC Laboratory Animal Co., Ltd. The animals were randomly divided into groups as needed, two pregnant mice for each group at every experiment. Antibody injection The purified and endotoxin-free $\mathrm{IgG}$ of the anti-coronavirus antibodies used for the animal model include rabbit polyclonal anti-COVID-19 S1, anti-COVID-19 N, anti-SARS-CoV S, mouse monoclonal anti- SARS-CoV N, and the human monoclonal anti-COVID-19 S1 or S-RBD antibodies of $\mathrm{B} 38^{15}$, REGN10987 $7^{17}, \mathrm{CC} 12.3^{18}$, and CR3022-B6 ${ }^{20}$. Two dosages of each antibody $\operatorname{IgG}$ were injected 
intraperitoneally $(I P)$ into timed-pregnant mice twice every three days at pregnancy (embryonic) day of E15 (about 26-28 g) and E18 (about 30-32 g) respectively (Figure 2A). For each polyclonal antibody, $50 \mu \mathrm{g}$ (microgram) for the first dose (about $2.0 \mathrm{mg} / \mathrm{kg}$ ) and 60 $\mu \mathrm{g}$ for the second dose (about $2.0 \mathrm{mg} / \mathrm{kg}$ ) were administrated. For each monoclonal antibody, $40 \mu \mathrm{g}$ for the first dose (about $1.5 \mathrm{mg} / \mathrm{kg}$ ) and $50 \mu \mathrm{g}$ for the second dose (about $1.5 \mathrm{mg} / \mathrm{kg}$ ) were administrated. In addition, the rabbit polyclonal antibody mixture consisted of 50 $\mu \mathrm{g}$ of the anti-COVID-19 $\mathrm{S} 1$ and $50 \mu \mathrm{g}$ of the anti-COVID-19 N, and the human monoclonal antibody mixture consisted of $40 \mu \mathrm{g}$ of the REGN10987, $20 \mu \mathrm{g}$ of the $\mathrm{CC} 12.3$, and $20 \mu \mathrm{g}$ of the CR3022-B6 were administered, respectively. The body weight of the pregnant mice was measured every day before and after the antibody injection. The mouse pups were born at about E20-E21 and the healthy status including clinical signs of the newborn mouse pups were observed and recorded (Figure 2B). The experiment was ended at day 1-2 post birth.

Sample collection At the end of the day, the blood samples were collected from newborn mouse pups, incubated at $4^{\circ} \mathrm{C}$ for overnight, centrifuged at $3000 \mathrm{rpm}$ for 5 minutes, and the supernatant was transferred to a new tube. The isolated sera were stored at $-80^{\circ} \mathrm{C}$ for cytokine detection. Lungs, hearts, brains, kidneys, livers, and intestines were collected from at least 3 mouse pups, fixed in formalin for $48-72$ hours, went through gradient alcohol dehydration and embedded in paraffin, and tissue sections were processed.

HE and immunofluorescent staining The mouse tissue sections of lungs, hearts, brains, kidneys, livers, and intestines were dewaxed and stained with hematoxylin-eosin (HE) for histology evaluation. For an immunofluorescent staining, the dewaxed sections were incubated at room temperature with the secondary antibodies of PE-labeled goat anti-human IgG (Abcam) or FITC-labeled goat anti-rabbit IgG (SouthernBiotech) for one hour, washed three times with PBS, and examined under an immunofluorescent microscope (Leica).

Tissue array Human tissue array slides consisted of various human fetal tissues (BE01015), or mutiple human diseased tissues of respiratory (LUD151), cardiovesvular (HE1001), urinary (BC07014) and digestive (BCC000128) system were purchased from US Biomax (Rockville, MD). 4-8 $\mu \mathrm{g}$ of biotin-labeled REGN10987 antibody was incubated with each of the tissues array slide at room temperature for one hour, washed off the free antibody, stained with FITC-labeled strepavidin for 30 minutes at room temperature, washed three times and examined under an immunofluorescent microscope (Leica).

\section{Acknowledgement}

We thank Dr. Haifeng Bao for his valuable advice on the development of the in vitro cellular assay, Dr. Xiaohui Zhou from Fudan University for reviewing the manuscript. The study was supported by funding from Hangzhou HuaAn PuChu Investment Limited Partnership.

\section{Competing Interests}

H.W. is the shareholder of Huirui Biopharma, X.L. and Y.C. are shareholders of HuaAn McAb Biotechnology, J.L. is a shareholder of Biolynx Technology. There are patent applications pending related to this work.

\section{References}

1. Lancet COVID-19 Commissioners, Task Force Chairs, and Commission Secretariat. Lancet COVID-19 Commission Statement on the occasion of the 75th session of the UN General Assembly. Lancet Lond. Engl. 396, 1102-1124 (2020).

2. Fauci, A. S., Lane, H. C. \& Redfield, R. R. Covid-19 - Navigating the Uncharted. N. Engl. J. Med. 382, 1268-1269 (2020).

3. Chen, J. et al. Clinical progression of patients with COVID-19 in Shanghai, China. J. Infect. 80, e1-e6 (2020).

4. Guan, W.-J. et al. Clinical Characteristics of Coronavirus Disease 2019 in China. N. Engl. J. Med. 382, 1708-1720 (2020).

5. Mokhtari, T. et al. COVID-19 and multiorgan failure: A narrative review on potential mechanisms. J. Mol. Histol. 51, 613-628 (2020).

6. Zhong, N. S. et al. Epidemiology and cause of severe acute respiratory syndrome (SARS) in Guangdong, 
People's Republic of China, in February, 2003. Lancet Lond. Engl. 362, 1353-1358 (2003).

7. Choi, W. S. et al. Clinical Presentation and Outcomes of Middle East Respiratory Syndrome in the Republic of Korea. Infect. Chemother. 48, 118-126 (2016).

8. Yu, W. et al. Characteristics of H7N9 avian influenza pneumonia: a retrospective analysis of 17 cases. Intern. Med. J. 50, 1115-1123 (2020).

9. Abdelrahman, Z., Li, M. \& Wang, X. Comparative Review of SARS-CoV-2, SARS-CoV, MERS-CoV, and Influenza A Respiratory Viruses. Front. Immunol. 11, 552909 (2020).

10. Melo-Vallès, A., Ballesté-Delpierre, C. \& Vila, J. Review of the Microbiological Diagnostic Approaches of COVID-19. Front. Public Health 9, 592500 (2021).

11. Wang, C. et al. Alveolar macrophage dysfunction and cytokine storm in the pathogenesis of two severe COVID-19 patients. EBioMedicine 57, 102833 (2020).

12. Fara, A., Mitrev, Z., Rosalia, R. A. \& Assas, B. M. Cytokine storm and COVID-19: a chronicle of pro-inflammatory cytokines. Open Biol. 10, 200160 (2020).

13. Sun, X. et al. Cytokine storm intervention in the early stages of COVID-19 pneumonia. Cytokine Growth Factor Rev. 53, 38-42 (2020).

14. Wang, H. Biological therapeutics for infectious or inflammatory diseases or conditions. PCT: WO2014/151526. (2014).

15. $\mathrm{Wu}, \mathrm{Y}$. et al. A noncompeting pair of human neutralizing antibodies block COVID-19 virus binding to its receptor ACE2. Science 368, 1274-1278 (2020).

16. Chi, X. et al. A neutralizing human antibody binds to the N-terminal domain of the Spike protein of SARS-CoV-2. Science 369, 650-655 (2020).

17. Hansen, J. et al. Studies in humanized mice and convalescent humans yield a SARS-CoV-2 antibody cocktail. Science 369, 1010-1014 (2020).

18. Yuan, M. et al. Structural basis of a shared antibody response to SARS-CoV-2. Science 369, 1119-1123 (2020).

19. Pinto, D. et al. Structural and functional analysis of a potent sarbecovirus neutralizing antibody. BioRxiv Prepr. Serv. Biol. (2020) doi:10.1101/2020.04.07.023903.

20. Rouet, R. et al. Potent SARS-CoV-2 binding and neutralization through maturation of iconic SARS-CoV-1 antibodies. http://biorxiv.org/lookup/doi/10.1101/2020.12.14.42 2791 (2020).

21. Huang, X. et al. Human Coronavirus HKU1 Spike Protein Uses $O$-Acetylated Sialic Acid as an Attachment Receptor Determinant and Employs Hemagglutinin-Esterase Protein as a Receptor-Destroying Enzyme. J. Virol. 89, 7202-7213 (2015).

22. Wang, S.-F. et al. Antibody-dependent SARS coronavirus infection is mediated by antibodies against spike proteins. Biochem. Biophys. Res. Commun. 451, 208-214 (2014).

23. Liu, L. et al. Anti-spike IgG causes severe acute lung injury by skewing macrophage responses during acute SARS-CoV infection. JCI Insight 4, (2019).

24. Liu, Y. et al. An infectivity-enhancing site on the SARS-CoV-2 spike protein targeted by antibodies. Cell S0092867421006620 (2021) doi:10.1016/j.cell.2021.05.032.

25. Schauer, R. \& Kamerling, J. P. Exploration of the Sialic Acid World. in Advances in Carbohydrate Chemistry and Biochemistry vol. 75 1-213 (Elsevier, 2018).

26. Marshall, M. The lasting misery of coronavirus long-haulers. Nature 585, 339-341 (2020).

27. Edwin O. Jordan. Epidemic Influenza: A survey. Chicago: American Medical Association. (1927).

28. Rose, N. R. Prediction and Prevention of Autoimmune Disease in the 21st Century: A Review and Preview. Am. J. Epidemiol. 183, 403-406 (2016). 


\section{Supplementary Files}

This is a list of supplementary files associated with this preprint. Click to download.

- FigureS1andS2.pdf 